\title{
LOSS ESTIMATION OF CAPACITOR IN HIGH REP-RATE PULSED POWER SYSTEM
}

\author{
Chaofeng Huang, Paul Melcher, George Ferguson and Richard Ness \\ Cymer, Inc. 17075 Thornmint Court \\ San Diego, CA 92127 USA
}

\begin{abstract}
Capacitor loss in pulsed power systems has become an important issue for thermal management, especially when the operating rep-rate and energy per pulse are getting higher and higher. It is practical to analyze the loss of a capacitor using a capacitor series equivalent circuit model in this pulsed power application. The capacitor loss is directly related to the equivalent series resistance (ESR) of the capacitor. In the paper, the formula to estimate the capacitor loss is presented. It is proportional to the energy per pulse, rep-rate, and capacitor dissipation factor $(\tan \delta)$ at the certain energy transfer frequency of the circuit. The charging and discharging frequencies of the capacitor might be different. Both of them will produce losses, and contribute to the total capacitor loss. The analysis of a capacitor bank with capacitors in parallel or in series is presented as well.
\end{abstract}

Also in this work, the parameters of different types of capacitors were measured, including film capacitors and high voltage ceramic capacitors, using a Agilent 4285A Precision LCR Meter up to $13 \mathrm{MHz}$. The influence of temperature on capacitance and dissipation factor was investigated by sinking the capacitor in oil under controlled temperatures. The results are very helpful to estimate the losses of capacitors in Cymer's laser pulsed power systems, and to provide design guidelines to the next generation of $6 \mathrm{kHz}$ laser power systems.

\section{I.INTRODUCTION}

In Cymer's laser pulsed power system, a high voltage IGBT is used as the switching device in the resonant energy transfer circuit from $\mathrm{C} 0$ to $\mathrm{C} 1$, and magnetic switches are used for resonant circuits from $\mathrm{C} 1$ to $\mathrm{Cp}-1$ and $\mathrm{Cp}-1$ to $\mathrm{Cp}$. The simplified schematic diagram of the Solid-State Pulsed Power Module (SSPPM) is shown in Figure 1.

$\mathrm{C} 0$ is charged by a resonant charger, and discharged into $\mathrm{C} 1$ by closing the IGBT switch. Then $\mathrm{C} 1$ discharges through a magnetic switch LS1 and a high voltage pulse transformer to $\mathrm{Cp}-1$. $\mathrm{Cp}-1$ discharges through magnetic switch LS2 to Cp and the laser chamber to generate laser light. Film capacitors are used for $\mathrm{C} 0$ and $\mathrm{C} 1$, and high voltage ceramic capacitors are used for $\mathrm{Cp}-1$ and $\mathrm{Cp}$.

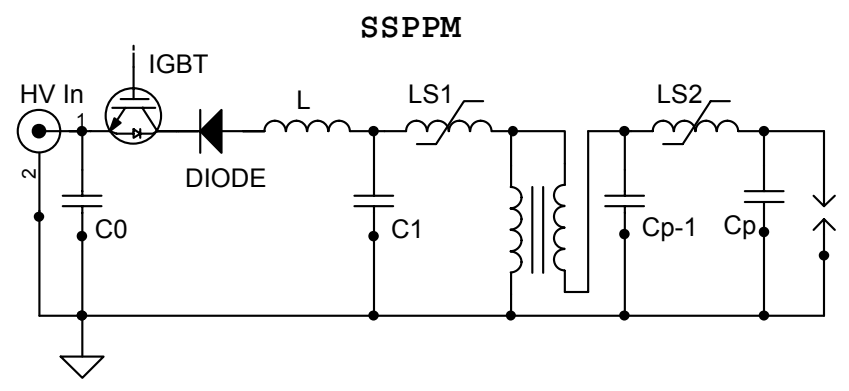

Figure 1. Simplified schematic diagram of SSPPM

The losses of the IGBT, series Diode and magnetic switch were studied before [1][2]. The analysis and test results presented in this paper are on the losses of capacitors as part of the SSPPM efficiency improvement and thermal management effort.

\section{II.CAPACITOR MODELS}

There are many different capacitor models. The two element and three element models are discussed here.

\section{A. Two Element Series Model}

The impedance vectors and capacitor series equivalent circuit model are shown in Figure 2.

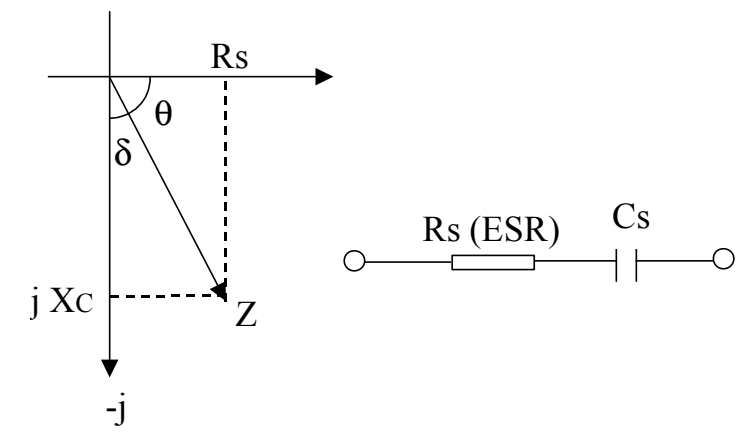

Figure 2. Capacitor two element series model

$Z$ : impedance; $\theta$ : phase angle

$\delta$ : the dielectric loss angle (complementary angle to $\theta$ )

$C s$ : equivalent series capacitance

$R s$ : equivalent series resistance (ESR)

$D$ : capacitor dissipation factor at energy transfer

frequency $(\tan \delta)$ 
The relationships of parameters are as follow.

$$
\begin{aligned}
& Z=R_{s}-j X_{C} ; X_{C}=\frac{1}{\omega C_{S}}=\frac{T}{2 \pi C_{S}} \\
& D=\tan \delta=\frac{R_{S}}{X_{C}}=R_{S} \bullet \omega C_{S} \\
& R_{S}=D \bullet X_{C}=\frac{D}{\omega C_{S}}=\frac{T \bullet \tan \delta}{2 \pi C_{S}}
\end{aligned}
$$

\section{B. Three Element Series Model}

Figure 3 is a three element series circuit model by taking into account the capacitor series equivalent inductance (Ls).

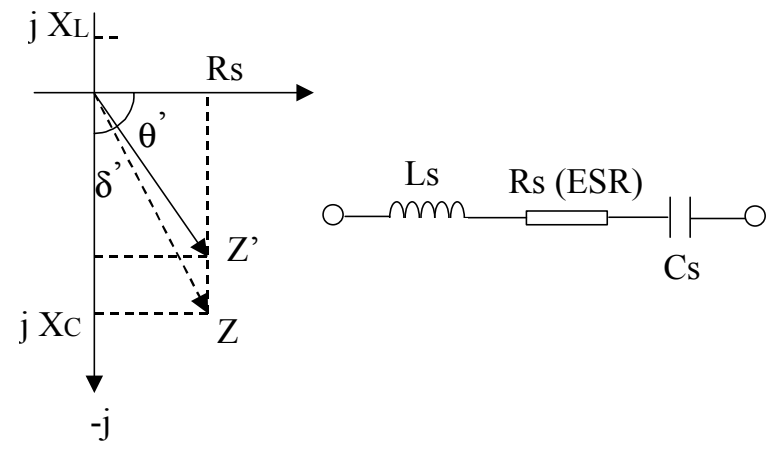

Figure 3. Capacitor three element series model

Rs is the most important parameter to consider for capacitor loss estimation. For the pulsed power system, the capacitor is usually used well below the self-resonant frequency $f=1 /\left(2 \pi \sqrt{L_{s} C_{s}}\right)$. Ls could be neglected here for our engineering application.

\section{CAPACITOR LOSS IN PULSED POWER CONDITION}

Considering the resonant energy transfer circuit from $\mathrm{C} 0$ to $\mathrm{C} 1$ in Figure 1 , the losses of $\mathrm{C} 0$ and $\mathrm{C} 1$ are estimated as follows. $\mathrm{C} 0$ is charged to $\mathrm{Vm}$, and discharged to $\mathrm{C} 1$ through inductor $\mathrm{L}$ by closing the IGBT (and assuming initial voltage on $\mathrm{Cl}$ is zero). The resistance of $\mathrm{C} 0, \mathrm{C} 1$ and IGBT and Diode are considered much smaller than the impedance of the charging inductor $\mathrm{L}$ and lumped capacitance.

The current would be a half sinusoidal waveform with period of $T$ and peak current of $I m$ as shown in Figure 4.

$$
\begin{aligned}
& I(t)=I_{m} \bullet \operatorname{Sin}(\omega t) \\
& \omega=\frac{2 \pi}{T}=\frac{1}{\sqrt{L \bullet C_{T}}} \\
& C_{T}=\frac{C_{0} \bullet C_{1}}{C_{0}+C_{1}} \\
& I_{m}=V_{m} \bullet \sqrt{\frac{C_{T}}{L}}=\frac{2 \pi \bullet V_{m} \bullet C_{T}}{T}
\end{aligned}
$$

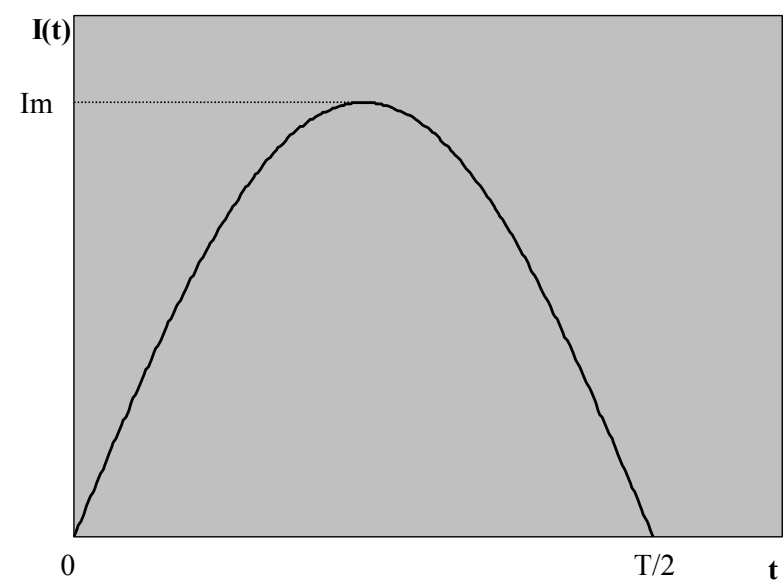

Figure 4. Current waveform from $\mathrm{C} 0$ to $\mathrm{C} 1$

Final voltage on $\mathrm{C} 0$ and $\mathrm{C} 1$ at end of charge $\mathrm{T} / 2$ :

$$
V_{C 0}=V_{m} \bullet\left(1-\frac{2 C_{1}}{C_{0}+C_{1}}\right) ; \quad V_{C 1}=V_{m} \bullet \frac{2 C_{0}}{C_{0}+C_{1}}
$$

Transferred energy per pulse:

$$
E=\frac{1}{2} \bullet C_{1} \bullet V_{C 1}{ }^{2}=2 \bullet V_{m}{ }^{2} \bullet \frac{C_{0}{ }^{2} \bullet C_{1}}{\left(C_{0}+C_{1}\right)^{2}}=\frac{2 \bullet V_{m}{ }^{2} \bullet C_{T}{ }^{2}}{C_{1}}
$$

The equivalent series resistance of $\mathrm{C} 0$ and $\mathrm{C} 1$ :

$$
R_{S 0}=\frac{T \bullet \tan \delta_{0}}{2 \pi C_{0}} ; \quad R_{S 1}=\frac{T \bullet \tan \delta_{1}}{2 \pi C_{1}}
$$

Energy loss on $\mathrm{C} 0$ per pulse:

$$
\begin{aligned}
& \Delta E_{0}=\int_{0}^{T / 2} R_{S 0} \bullet I(t)^{2} \bullet d t=R_{S 0} \bullet I_{m}{ }^{2} \bullet \int_{0}^{T / 2} \operatorname{Sin}^{2}(\omega t) \bullet d t \\
& =\frac{R_{S 0} \bullet I_{m}{ }^{2} \bullet T}{4}=\frac{\pi}{2} \bullet \tan \delta_{0} \bullet \frac{V_{m}{ }^{2} \bullet C_{T}{ }^{2}}{C_{0}} \\
& =\frac{\pi}{4} \bullet \tan \delta_{0} \cdot \frac{C_{1}}{C_{0}} \bullet E
\end{aligned}
$$

Energy loss on $\mathrm{C} 1$ per pulse:

$$
\begin{aligned}
& \Delta E_{1}=\int_{0}^{T / 2} R_{S 1} \bullet I(t)^{2} \bullet d t=\frac{\pi}{2} \bullet \tan \delta_{1} \bullet \frac{V_{m}{ }^{2} \cdot C_{T}{ }^{2}}{C_{1}} \\
& =\frac{\pi}{4} \bullet \tan \delta_{1} \bullet E
\end{aligned}
$$

$\mathrm{C} 0$ discharging loss (power dissipation) at pulsed reprate condition:

$$
C_{0} \text { Loss }=\frac{\pi}{4} \bullet \tan \delta_{0} \bullet \frac{C_{1}}{C_{0}} \bullet E \bullet(\text { rep }- \text { rate })
$$

$\mathrm{C} 1$ charging loss (power dissipation) at pulsed rep-rate condition:

$$
C_{1} \text { Loss }=\frac{\pi}{4} \bullet \tan \delta_{1} \bullet E \bullet(r e p-r a t e)
$$


One can see from formula (1) and (2) that the capacitor loss at pulsed power condition is proportional to the capacitor dissipation factor, transferred energy and the pulse rep-rate. Also the loss formulas are different between discharging and charging capacitors if their capacitances are different.

The formulas are also applicable to the $\mathrm{C} 1 / \mathrm{Cp}-1$, and Cp-1 / Cp energy transfer loops.

\section{CAPACITOR BANK}

Usually, the capacitor bank used for $\mathrm{C} 0, \mathrm{C} 1, \mathrm{Cp}-1$, or $\mathrm{Cp}$ consists of the same individual capacitors in a parallel and/or series array.

\section{A. Capacitors in Parallel}

Figure 5 shows a number of $\mathrm{n}$ capacitors in parallel and the equivalent circuit.

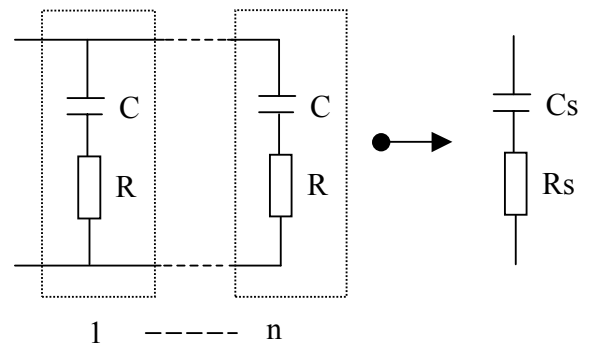

Figure 5. Capacitors in parallel

$$
\begin{aligned}
& C_{S}=n \bullet C ; \quad R_{S}=R / n \\
& \tan \delta_{S}=R_{S} \bullet \omega C_{S}=R \bullet \omega C=\tan \delta
\end{aligned}
$$

One can see that the dissipation factor of the capacitor bank with parallel capacitors equals to that of the single capacitor.

\section{B. Capacitors in Series}

Figure 6 shows a number of $\mathrm{n}$ capacitors in series and the equivalent circuit.

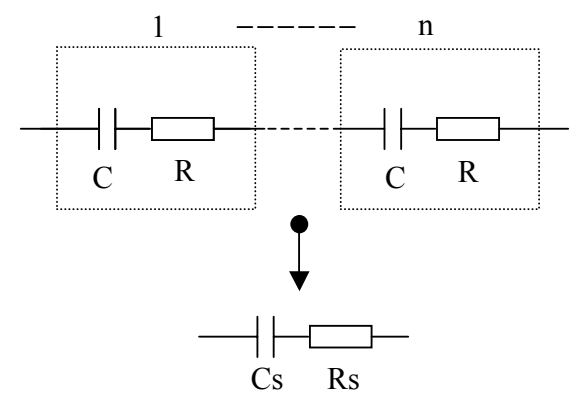

Figure 6. Capacitors in series

$C_{S}=C / n ; \quad R_{S}=n \bullet R$

$\tan \delta_{S}=R_{S} \bullet \omega C_{S}=R \bullet \omega C=\tan \delta$

One can see that the dissipation factor of the capacitor bank with series capacitors equals to that of the single capacitor.
If a capacitor bank consists of same capacitors in parallel or series array, the dissipation factor of a single capacitor can be used to calculate the whole capacitor bank loss by formulas (1) or (2).

\section{CAPACITOR MEASUREMENT}

An Agilent 4285A Precision LCR Meter was used to measure the parameters of polypropylene film capacitor and high voltage ceramic capacitor samples.

\section{A. Film capacitor $0.047 u F, 2000 \mathrm{VDC}$}

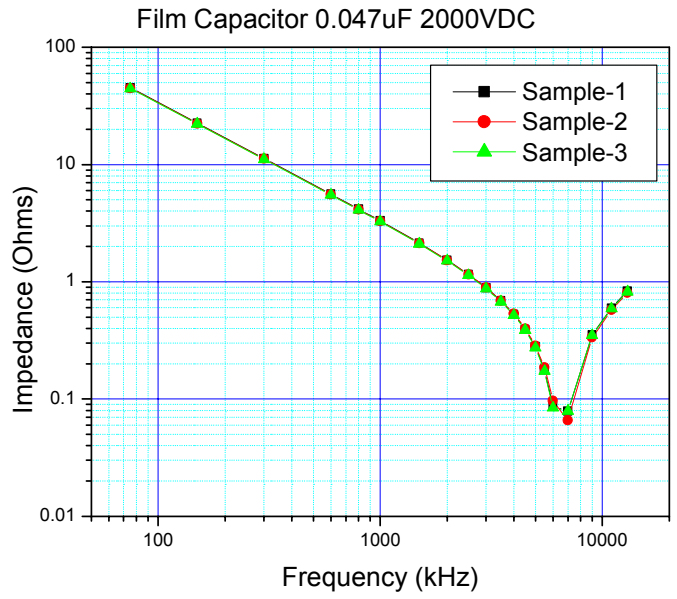

Figure 7. The impedance changing with frequency

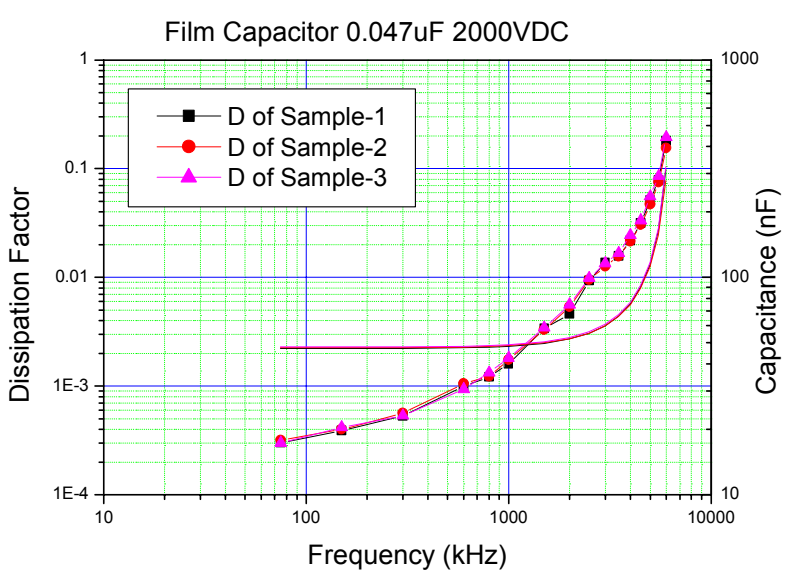

Figure 8. Capacitance and dissipation factor vs. frequency

Figure 7 shows the measured impedance changing with signal frequency. The capacitive reactance dominates at the low frequency range (less than $7 \mathrm{MHz}$ ) and the impedance decreases with increasing frequency. At the high frequency range (greater than $7 \mathrm{MHz}$ ), the inductive reactance dominates. The self-resonant frequency is about $7 \mathrm{MHz}$. 
Figure 8 shows the measured capacitance and dissipation factor changing with frequency.

\section{B. High voltage ceramic capacitor $390 \mathrm{pF}, 50 \mathrm{kV}$}

Figure 9 shows the measured impedance changing with frequency up to $13 \mathrm{MHz}$, which is the limit of the Test Fixture. The ceramic capacitor self-resonant frequency is beyond $13 \mathrm{MHz}$.

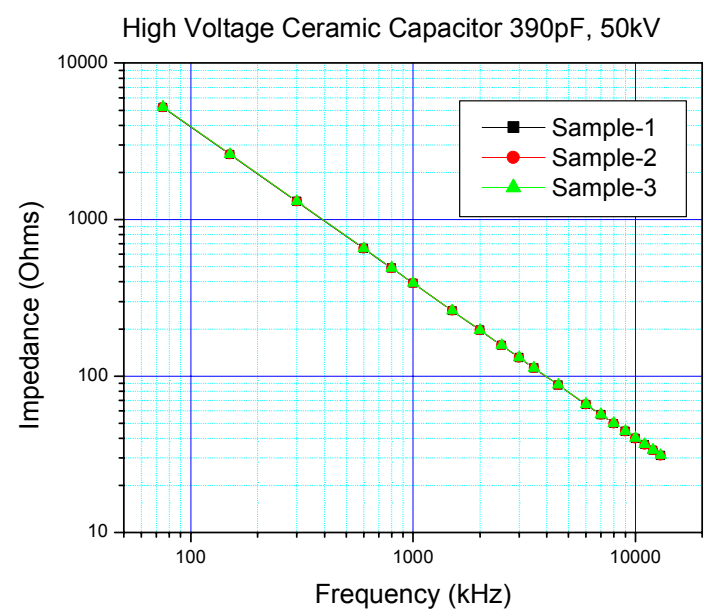

Figure 9. The impedance changing with frequency

Figure 10 shows the measured capacitance and dissipation factor changing with frequency.

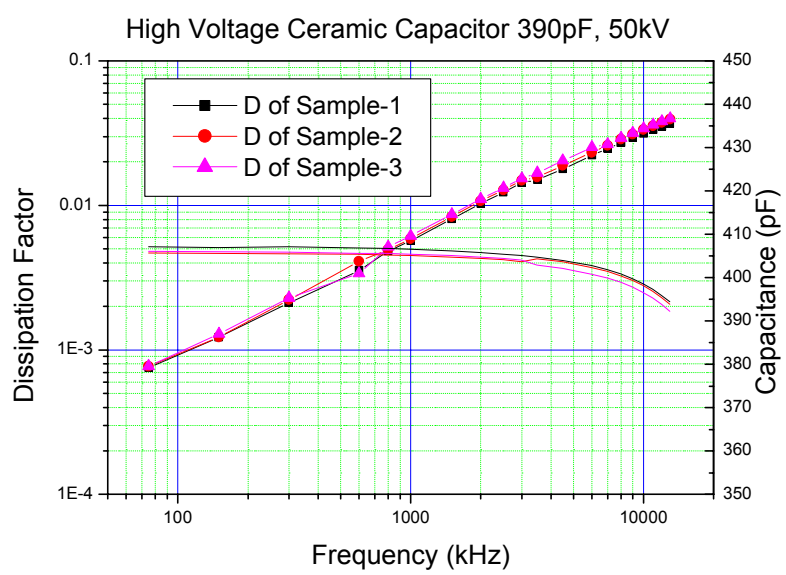

Figure 10. Capacitance and dissipation factor vs. frequency

\section{Temperature influence on capacitors}

The temperature influence on capacitance was measured by sinking the capacitors in oil under controlled temperature. Figure 11 shows the capacitance changing with temperatures at $1 \mathrm{MHz}$. One can see that the temperature coefficient of the ceramic capacitor (4220PPM) is much larger than the film capacitor (365PPM).

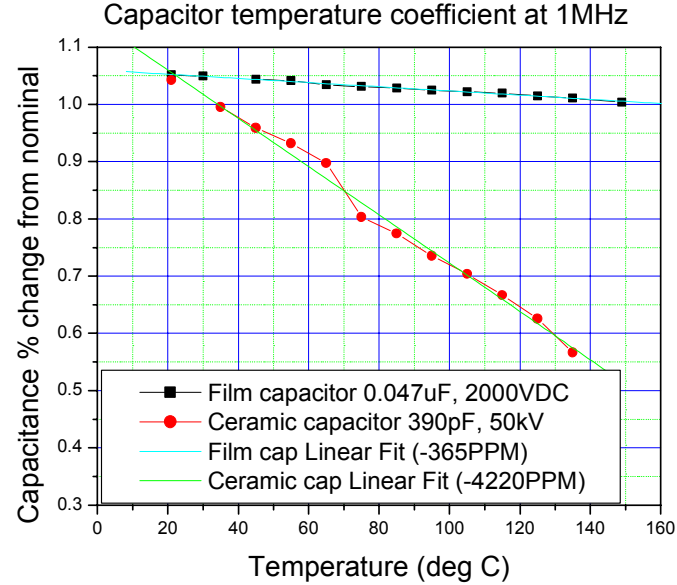

Figure 11. Capacitance change vs. temperature at $1 \mathrm{MHz}$

\section{One example of capacitor Cp-1 loss estimation}

Assuming $\mathrm{C} 1=\mathrm{Cp}-1=\mathrm{Cp}, \mathrm{Cp}-1$ consists of 20 pieces of $390 \mathrm{pF}$ ceramic capacitors, energy transfer on $\mathrm{Cp}-1$ is $5.0 \mathrm{~J} /$ pulse, rep-rate is $6 \mathrm{kHz}, \mathrm{Cp}-1$ charging time is $333 \mathrm{~ns}$, ( $\mathrm{D}=8.0 \mathrm{E}-3$ at $1.5 \mathrm{MHz}$ from Figure 10), discharge time is $71.4 \mathrm{~ns}(\mathrm{D}=0.025$ at $7 \mathrm{MHz}$ from Figure 10$)$.

$C_{p-1}$ Total Loss $=C_{p-1}$ Ch $\arg$ e Loss $+C_{p-1}$ Disch $\arg$ e Loss $=\frac{\pi}{4} \times(8.0 E-3+0.025) \times 5.0 \times 6000=777.5(\mathrm{~W})$

\section{SUMMARY}

The capacitor loss in pulsed power rep-rate condition is analyzed in the paper. And also the parameters of capacitors were measured. The following conclusions are observed:

- The capacitor charging or discharging loss is proportional to the dissipation factor, energy transfer per pulse, and the pulse rep-rate.

- The dissipation factor of a capacitor bank consisting of same capacitors in parallel or series array is the same as an individual capacitor.

- The dissipation factors of the film capacitor and ceramic capacitors are increasing with signal frequency. Measured capacitance of the film capacitor increases with frequency; capacitance of the ceramic capacitor decreases with frequency.

\section{REFERENCES}

[1] Chaofeng Huang, Paul Melcher, George Ferguson and Richard Ness, "IGBT and Diode Loss Measurements in Pulsed Power Operating Conditions", 2004 Power Modulator Conference, San Francisco, May 23-26, 2004.

[2] Kenneth McDonald, Randy Curry, Renuka Narsetti, Richard Ness, Paul Melcher, and Chaofeng Huang, "Evaluation of Magnetic Materials and Insulation Systems for Repetition-Rate Pulse Compression Applications", $14^{\text {th }}$ IEEE International Pulsed Power Conference, Dallas, Texas, USA, June 15-18, 2003. 